\title{
Self-phase modulation and modal noise in optical fibers
}

\author{
Bruno Crosignani \\ Fondazione Ugo Bordoni, Istituto Superiore P.T., Viale Europa 160, Roma, Italy \\ Paolo Di Porto \\ Istituto di Fisica, Università dell' Aquila, L'Aquila, Italy, and Fondazione Ugo Bordoni
}

Received March 4, 1982; revised manuscript received June 5, 1982

\begin{abstract}
The phase variations associated with the intensity-dependent part of the refractive index assume different values for the different propagation modes of an optical fiber. As a consequence, intensity fluctuations of the exciting source are converted into relative phase fluctuations, which give rise to an amplitude-dependent modal noise.
\end{abstract}

\section{INTRODUCTION}

The study of the influence of the intensity-dependent part of the refractive index on the propagation of an optical pulse reveals the existence of a self-focusing mechanism ${ }^{1}$ and of a self-phase modulation. ${ }^{2}$ The latter effect, consisting of a modulation of the phase of the field proportional to its instantaneous intensity, is particularly relevant in an optical fiber because of the long interaction length provided by the guiding structure and has actually been observed in singlemode fibers. ${ }^{3}$ In a multimode fiber, the phase modulation of each modal field will depend on the spatial configuration of the mode itself, the effect being larger, for equal modal powers, for modes possessing a smaller effective area. Accordingly, the nonlinear contribution to the phases of different modes will be different, so that a fluctuation of the relative phase will arise between any pair of modes as a consequence of fluctuations present in the amplitude of the signal.

In turn, this represents a nonlinear contribution to standard modal noise, which is a linear phenomenon usually associated with phase fluctuations that are due to frequency fluctuations of the input signal. 4 We present in the following an analytical description of the effect. The analysis of propagation in a multimode optical fiber in the presence of an intensity-dependent refractive index shows that the dephasing of each mode is due to its nonlinear interaction both with itself (self-phase modulation) and with the other modes. The effect turns out to be appreciable for a multimode glass fiber $1 \mathrm{~km}$ long, when an input power corresponding to few milliwatts per mode is employed.

\section{ANALYTICAL DESCRIPTION}

The approach adopted in this section is closely related to the one already developed for describing soliton propagation in multimode optical fibers, ${ }^{5}$ which is essentially related to the mutual competition between self-phase modulation and chromatic dispersion. 6,7 Our description is based on the coupled-mode formalism first introduced in the frame of fiber optics for treating linear propagation in the presence of mode coupling associated with fiber imperfections. ${ }^{8}$ In the present context, coupling is induced by the nonlinear contribution to the refractive index

$$
n=n_{1}+n_{2}|\hat{\mathbf{E}}|^{2},
$$

where $n_{1}$ is the linear refractive index and $\hat{\mathbf{E}}$ is the analytic signal of the electric field. The term $n_{2}|\hat{\mathbf{E}}|^{2}$ formally plays the role of fiber imperfection. Actually, the relation between the third-order nonlinear susceptibility $\chi^{(3)}$ and the electric field E

$$
\mathbf{P}^{(3)}=\epsilon_{0} \chi^{(3)}(\mathbf{E} \cdot \mathbf{E}) \mathbf{E},
$$

( $\epsilon_{0}$ being the vacuum permeability) implies Eq. (1) only if the field possesses an initial linear polarization preserved along the fiber. In general, the nonlinear effect is described by the following tensorial relation between the Fourier transforms at the angular frequency $\omega$ of the transverse part of the electric displacement vector $\mathbf{D}$ and of the electric vector $\mathbf{E}$ (the longitudinal $z$ components are negligible in weakly guiding fibers possessing a cylindrical symmetry around the $z$ axis):

$$
\mathbf{D}_{T \omega}=\epsilon_{1}(\omega) \mathbf{E}_{T \omega}+\epsilon_{T}^{(3)}: \mathbf{E}_{T \omega},
$$

where

$$
\begin{aligned}
\epsilon_{T}^{(3)} & =\frac{\epsilon_{0} \chi^{(3)}}{2} \\
& \times\left|\begin{array}{ll}
\hat{\mathbf{E}}_{T} \times \hat{\mathbf{E}}_{T}{ }^{*}+1 / 2\left|\hat{E}_{x}\right|^{2}, & 1 / 2 \hat{E}_{y} \hat{E}_{x}{ }^{*} \\
1 / 2 \hat{E}_{x} \hat{E}_{y} * & \hat{\mathbf{E}}_{T} \times \hat{\mathbf{E}}_{T}^{*}+1 / 2\left|\hat{E}_{y}\right|^{2}
\end{array}\right|
\end{aligned}
$$

and $\epsilon_{1}=\epsilon_{0} n_{1}^{2}$.

In actual multimode optical fibers, in which polarization is completely scrambled over few meters of length, the nondiagonal terms of the matrix appearing in Eq. (4) can be assumed to be zero, and, one takes advantage of the relation $\left|\hat{E}_{x}\right|^{2}=\left|\hat{E}_{y}\right|^{2}=1 / 2\left|\hat{\mathbf{E}}_{T}\right|^{2}$, Eq. (1) still applies with $n_{2}=$ $5 \chi^{(3)} / 16 n_{1}$ [for a linearly polarized field one has $n_{2}=3 \chi^{(3)} /$ $\left.8 n_{1}\right]$.

The analytic signal of the field propagating inside the fiber can be expanded in the form

$$
\begin{aligned}
\hat{\mathbf{E}}_{T}(\mathbf{r}, z) & =\sum_{m} \mathbf{E}_{m}(\mathbf{r}) \int_{0}^{+\infty} c_{m}(z, \omega) \exp \left[i \omega t-i \beta_{m}(\omega) z\right] \mathrm{d} \omega \\
& \equiv \sum_{m} \mathbf{E}_{m}(\mathbf{r}) \exp \left[i \omega_{0} t-i \beta_{m}\left(\omega_{0}\right) z\right] \hat{\Phi}_{m}(z, t),
\end{aligned}
$$

where $\mathbf{E}_{m}(\mathbf{r})$ and $\beta_{m}(\omega)$ represent the spatial configuration and the propagation constant of the $m$ th mode, respectively, 
$\mathbf{r}=(x, y)$ is the transverse coordinate, and $\omega_{0}$ is the midfrequency of the field.

According to the results of Ref. 5, the slowly varying amplitudes $\hat{\Phi}_{m}(z, t)$ obey (neglecting fiber chromatic dispersion) the set of nonlinear differential equations

$$
\begin{aligned}
&\left(\frac{\partial}{\partial z}+\frac{1}{V_{m}} \frac{\partial}{\partial t}\right) \hat{\Phi}_{m}= \\
&-2 i \hat{\Phi}_{m}\left\{\sum_{n \neq m} R_{n m}\left|\hat{\Phi}_{n}\right|^{2}+1 / 2 R_{m m}\left|\hat{\Phi}_{m}\right|^{2}\right\}, \\
& \quad m=1,2, \ldots,
\end{aligned}
$$

where $V_{m}=\left(\mathrm{d} \beta_{m} / \mathrm{d} \omega\right)_{\omega=\omega_{0}}{ }^{-1}$ is the group velocity of the $m$ th mode and

$$
R_{n m}=\frac{\omega_{0} n_{2}}{c} \frac{\iint_{-\infty}^{+\infty} E_{n}^{2}(\mathbf{r}) E_{m}^{2}(\mathbf{r}) \mathrm{d} x \mathrm{~d} y}{\iint_{-\infty}^{+\infty} E_{m}^{2}(\mathbf{r}) \mathrm{d} x \mathrm{~d} y} .
$$

The set of Eqs. (6) can easily be integrated after introducing the new variables $z^{\prime}=z$ and $t_{m}{ }^{\prime}=t-z / V_{m}$, if we observe that the structure of the set is such that

$$
\left|\hat{\Phi}_{m}(z, t)\right|^{2}=\left|\hat{\Phi}_{m}\left(z=0, t-z / V_{m}\right)\right|^{2}, \quad m=1,2, \ldots
$$

By taking advantage of this fact, we obtain

$$
\begin{aligned}
& \hat{\Phi}_{m}(z, t)=\hat{\Phi}_{m}\left(z=0, t-z / V_{m}\right) \\
& \times \exp \left\{-2 i \sum_{n \neq m} R_{n m}\right. \\
&\left.\times \int_{0}^{z}\left|\hat{\Phi}_{n}\left[z=0, t-z^{\prime \prime} / V_{n}+\left(z^{\prime \prime}-z\right) / V_{m}\right]\right|^{2} \mathrm{~d} z^{\prime \prime}\right\} \\
& \times \exp \left[-i R_{m m}\left|\hat{\Phi}_{m}\left(z=0, t-z / V_{m}\right)\right|^{2} z\right], \\
& m=1,2, \ldots
\end{aligned}
$$

The set of Eqs. (9) shows that the phase of each mode contains nonlinear contributions proportional to the powers carried by the various modes and that will exhibit, as a consequence, temporal variations strictly connected to the ones undergone by the modal powers. Thus, if the amplitude of the input signal fluctuates, each mode field acquires, besides an obvious amplitude modulation, a phase modulation that adds to that induced by the frequency fluctuations of the source.

\section{NONLINEAR MODAL NOISE}

Modal noise is associated with the presence of interference among the various guided modes of the fiber, and its behavior will accordingly depend on the quantities $\hat{\Phi}_{m} \hat{\Phi}_{l} *$. In order to give an estimate of the change induced by the nonlinearity on the phase of these quantities, let us assume that all the modes possess the same power, and let us neglect their relative mode delays. Under these assumptions, the typical nonlinear contribution $\Gamma_{\mathrm{NL}}$ to the phase of $\hat{\Phi}_{m} \hat{\Phi}_{l} *$ is of the kind

$$
\Gamma_{\mathrm{NL}}=\left(R_{l l}-R_{m m}\right)|\hat{\Phi}|^{2} z,
$$

where $|\hat{\Phi}|^{2}$ represents the common value of the $\left|\hat{\Psi}_{m}\right|^{2}$ 's.

By recalling Eq. (7) and assuming that the modes are normalized,

$$
\iint_{-\infty}^{+\infty} E_{m}^{2}(\mathbf{r}) \mathrm{d} x \mathrm{~d} y=1
$$

we have

$$
\Gamma_{\mathrm{NL}}=2 k\left(n_{2} / n_{1}\right) Z_{0} P\left(1 / A_{l}-1 / A_{m}\right) z,
$$

where $k=\omega_{0} / c, P$ is the power carried by each mode, $Z_{0}$ is the vacuum impedance, and $A_{l}$ is the effective area ${ }^{3}$ of the $l$ th mode. In order to evaluate $A_{l}$, let us write

$$
E_{l}^{2}(\mathbf{r})=\frac{F_{l}\left(\chi_{l} r\right)}{\iint_{-\infty}^{+\infty} F_{l}\left(\chi_{l} r\right) \mathrm{d} x \mathrm{~d} y},
$$

with $\chi_{l}=\left(n_{1}^{2} k^{2}-\beta_{l}^{2}\right)^{1 / 2}$. One then has

$$
\frac{1}{A_{l}}=\chi_{l}^{2} g_{l} / 2 \pi
$$

where

$$
g_{l}=\frac{\int_{0}^{+\infty} F_{l}^{2}(\rho) \rho \mathrm{d} \rho}{\left[\int_{0}^{+\infty} F_{l}(\rho) \rho \mathrm{d} \rho\right]^{2}} .
$$

If the $g_{l}$ 's pertaining to different modes are not too dissimilar ( $g_{l}=1$ if $F_{l}$ is Gaussian), we can finally write

$$
\Gamma_{\mathrm{NL}}=\left(k n_{2} / \pi n_{1}\right) Z_{0} P\left(\chi_{l}^{2}-\chi_{m}^{2}\right) z \cong(2 / \pi) k^{2} n_{2} Z_{0} P \delta \beta z,
$$

where $\delta \beta=\beta_{l}-\beta_{m}$. Assuming that the difference $\delta \beta$ between the propagation constants to be the average one pertaining to the modes of a fiber possessing a normalized refractive-index difference $\Delta$, one has $\delta \beta \cong \Delta^{1 / 2} / a$. By choosing, for example, $\lambda=0.8 \mu \mathrm{m}, n_{2} \cong 3 \times 10^{-22} \mathrm{~m}^{2} / \mathrm{V}^{2}, \Delta=10^{-2}$, and $z=10^{3} / \mathrm{m}$, a nonlinear phase variation of the order of unity is obtained for $P$ of the order of a few milliwatts and $a$, the fiber radius, of the order of a few micrometers.

\section{CONCLUSIONS}

We have evaluated the variation of the phase differences among the modes of an optical fiber induced by the presence of an intensity-dependent refractive index. More precisely, a variation of the signal amplitude affects the relative phase of the various modes and thus the configuration of the speckle pattern on a given fiber section. As a consequence, inputpower fluctuations give rise to a fluctuation of the speckle pattern, which can be interpreted ${ }^{9,10}$ as a kind of nonlinear modal noise.

We wish finally to note that, although we have considered a case in which chromatic dispersion is negligible, the validity of our results extends in practice to all situations in which the pulse duration exceeds the chromatic delay. ${ }^{11}$

\section{REFERENCES}

1. R. Y. Chiao, E. Garmire, and C. H. Townes, "Self-trapping of optical beams," Phys. Rev. Lett. 13, 479-482 (1964).

2. F. Shimizu, "Frequency broadening in liquids by a short light pulse," Phys. Rev. Lett. 19, 1097-1100 (1967).

3. R. H. Stolen and C. Jin, "Self-phase modulation in silica optical fibers," Phys. Rev. A 17, 1448-1453 (1978).

4. R. E. Epworth, "Modal noise-causes and cures," Laser Focus 17(9), 109-115 (1981). 
5. B. Crosignani and P. Di Porto, "Soliton propagation in multimode optical fibers," Opt. Lett. 6, 329-330 (1981).

6. A. Hasegawa and F. Tappert, "Transmission of stationary nonlinear optical pulses in dispersive dielectric fibers. I. Anomalous dispersion," Appl. Phys. Lett. 23, 142-144 (1973).

7. A. Hasegawa, "Self-confinement of multimode optical pulses in a glass fiber," Opt. Lett. 5, 416-417 (1980).

8. D. Marcuse, Theory of Dielectric Optical Waveguides (Academic, New York, 1974).
9. B. Daino, G. De Marchis, and S. Piazzolla, "Speckle and modal noise in optical fibres. Theory and experiment," Opt. Acta 27, 1151-1159 (1980).

10. E. G. Rawson, J. W. Goodman, and R. E. Norton, "Frequency dependence of modal noise in multimode optical fibers," J. Opt. Soc. Am. 70, 968-976 (1980).

11. B. Crosignani and P. Di Porto, "Degree of polarization in a birefringent single-mode optical fibre," Electron. Lett. 18, 15-16 (1982). 